\section{ANOS DO SERVIÇO DE ORIENTAÇÃO AO EXERCÍCIO EM VITÓRIA/ES: PIONEIRISMO NAS PRÁTICAS CORPORAIS E ATIVIDADES FÍSICAS NO SISTEMA ÚNICO DE SAÚDE}

\author{
30 YEARS OF EXERCISE GUIDANCE IN VITORIA: A PIONEERING \\ SERVICE IN BODY PRACTICES AND PHYSICAL ACTIVITIES UNDER \\ THE UNIFIED HEALTH SYSTEM C
}

30 AÑOS DEL SERVICIO DE ORIENTACIÓN AL EJERCICIO EN VITÓRIA/ ES: PIONERISMO EN LAS PRÁCTICAS CORPORALES Y ACTIVIDADES FÍSICAS EN EL SISTEMA ÚNICO DE SALUD C P

doi' https://doi.org/10.22456/1982-8918.103142

\section{Leonardo Araújo Vieira* <|caramuru@gmail.com>}

Sabrina Barbosa Garcia de Albuquerque* <sbgalbuquerque@gmail.com> Fábio Olímpio Venturim*<fabioventurim@yahoo.com.br>

Fabio Fortunato Brasil de Carvalho** <fabiofbcarvalho@gmail.com>

(i) Ueberson Ribeiro Almeida*** <uebersonribeiro@hotmail.com>

\footnotetext{
*Prefeitura Municipal de Vitória. Vitória, ES, Brasil.

**Instituto Nacional de Câncer José Alencar Gomes da Silva (INCA), Ministério da Saúde. Rio de Janeiro, RJ, Brasil.

***Universidade Federal do Espírito Santo. Vitória, ES, Brasil.
}

Resumo: O texto analisa o pioneirismo do Serviço de Orientação ao Exercício (SOE) de Vitória/ES a partir da promoção das práticas corporais e atividades físicas (PCAF) no Sistema Único de Saúde (SUS). Trata-se de uma estratégia coletiva de reflexão e escrita tecida pelos profissionais, gestores do programa e pesquisadores parceiros vinculados à Educação Física e à Saúde Coletiva. Faz um balanço e discute as principais transformações ocorridas no SOE ao longo de seus 30 anos. Apresenta como o Serviço vem dialogando, ao longo dessas três décadas, com as políticas de saúde e com a literatura acerca da atividade física e das PCAF. Identifica potencialidades e desafios do SOE como programa de saúde pública, bem como destaca seu protagonismo no campo das PCAF no SUS. Afirma a importância da articulação e diálogo com outras instituições, políticas, programas e práticas de saúde para a qualificação do serviço que o SOE oferta à população.

Palavras chave: Política de Saúde. Atividade Motora. Sistemas de Saúde. Colaboração Intersetorial.
Recebido em: 19-05-2020 Aprovado em: 25-10-2020 Publicado em: 09-12-2020

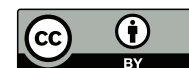

Este é um artigo publicado sob a licença Creative Commons Atribuição 4.0 Internacional (CC BY 4.0). elSSN: 1982-8918 


\section{INTRODUÇÃO}

As práticas corporais e atividades físicas $\left(\mathrm{PCAF}^{1}\right)$ possuem relevância e reconhecimento na promoção e cuidado à saúde, com importantes posicionamentos recentes de instituições como a Organização Mundial da Saúde (WHO, 2018, 2020) e o Programa das Nações Unidas para o Desenvolvimento (PNUD, 2017). No Sistema Único de Saúde (SUS), as PCAF encontram-se no discurso e nas ações governamentais, tanto no Ministério da Saúde (CARVALHO, 2020) quanto na esfera municipal.

O Serviço de Orientação ao Exercício (SOE) de Vitória, capital do estado do Espírito Santo, por exemplo, é um programa de promoção das PCAF da Secretaria Municipal de Saúde (Semus), implantado em 1990, completando, portanto, 30 anos de atividade em 2020.

Nesse contexto, parte-se do pressuposto de que o SOE foi aperfeiçoado ao longo de 30 anos de trajetória, influenciado por modificações nas políticas de saúde. Como programa contemporâneo à criação do SUS, repercutiu na implantação de programas similares em outros municípios, estados e em âmbito nacional. Diante disso, cabe destacar que o SOE foi considerado como um modelo pela Sociedade Brasileira de Medicina do Esporte (REZENDE, 1997). Além disso, o SOE é reconhecido pelo Ministério da Saúde como iniciativa pioneira e como um dos programas municipais que serviram como uma fonte de inspiração para a criação do Programa Academia da Saúde (BRASIL, 2019a).

Assim, este é um momento oportuno e importante (do ponto de vista da "maturidade" do SOE) para apresentar as experiências acumuladas ao longo desses 30 anos. Este ensaio propõe-se a resgatar e analisar parte da história do programa com vistas a evidenciar o pioneirismo nas PCAF no SUS, destacando alguns pontos marcantes que implicaram modificações nos modos de gestão e organização do serviço e, com efeito, na qualificação do seu funcionamento como programa de saúde pública.

Os referidos resgates e análises perpassarão por algumas evidências sobre a potencialidade do serviço, pelo destaque de alguns desafios e, por fim, por apontamentos de perspectivas futuras e considerações sobre a importância do fortalecimento do SOE, do Programa Academia da Saúde e das ações de PCAF no SUS. Para tanto, são utilizadas como fontes, em uma estratégia coletiva de reflexão e escrita, experiências vividas pelos autores, profissionais e gestores do SOE, sujeitos diretamente implicados na referida história, e por pesquisadores da Educação Física e Saúde Coletiva, imbricando "olhares internos e externos" na construção do texto, o qual possui caráter ensaístico.

\section{UM BREVE HISTÓRICO SOBRE O SOE}

Apresentar o histórico do SOE, estabelecendo marcos que determinaram mudanças em sua organização e funcionamento ao longo de três décadas, é uma

\footnotetext{
1 Neste texto as práticas corporais e atividades físicas (PCAF) serão abordadas em conjunto por compreendermos que estes termos são complementares e igualmente importantes para o campo da Educação Física e Saúde, apesar de reconhecermos que existe um importante debate conceitual no uso dos termos. Entretanto, quando se tratar de uma citação que aborde um dos termos/conceitos, isso será respeitado.
} 
tarefa complexa e, ao mesmo tempo, uma oportunidade singular, ainda pouco explorada, para conexões com a história das PCAF no SUS, suas relações com as políticas públicas de saúde e com a Educação Física no Brasil.

A criação do SOE se estabelece por meio de legislação municipal em 1985. Inicialmente, foi denominado como Serviço de Orientação à Fisiologia do Exercício (Sorfe). Teve seu primeiro módulo (piloto) implantado em 1990, com o objetivo de orientar a prática de exercício para a população em geral que espontaneamente se exercitava na orla de Vitória/ES. Em seu projeto inicial, o SOE tinha a proposta de se consolidar como um programa para combater o sedentarismo por meio da massificação da atividade física, pautando-se nas discussões do campo que enfatizam os benefícios do exercício físico para a prevenção de doenças, sobretudo das doenças crônicas não transmissíveis (DCNT), mas também sinalizando para outros objetivos voltados para possibilidades ampliadas de compreensão da atividade física na saúde, ainda que em caráter incipiente.

Nos primeiros anos de funcionamento, a equipe que compunha o SOE foi constituída por professores de Educação Física cedidos pela Secretaria Municipal de Educação, e contava com a participação de acadêmicos de Medicina, Enfermagem e Educação Física. Cabe ressaltar que, inicialmente, o ingresso no SOE era condicionado à realização de um curso de Fisiologia do Exercício oferecido pela Prefeitura de Vitória em parceria com a Universidade Federal do Espírito Santo (UFES).

Dessa forma, é possível perceber que a interdisciplinaridade e a intersetorialidade são algumas características presentes desde o início do funcionamento do programa. Entretanto, embora se reconheça a importância da Fisiologia do Exercício para a formação de profissionais da saúde, em especial para os de Educação Física, a obrigatoriedade desse curso em específico para atuar no SOE demonstra a predominância dos aspectos biológicos, o que é hegemônico no campo da saúde e, consequentemente, em programas de promoção das PCAF no SUS, sendo possível afirmar que, de alguma forma, isto ainda é presente na atualidade.

Ao longo da primeira década de funcionamento (1990 a 2000), o SOE foi ampliado por meio da instalação de novos módulos em praças, parques e praias do município, e pelo aumento do número de profissionais do serviço. Essas mudanças estruturais foram importantes para a ampliação do acesso ao serviço pelas comunidades e abriram um caminho para a promoção das PCAF na saúde pública e como campo de atuação de profissionais de Educação Física no SUS. Apesar disso, a consolidação do SOE como uma política pública estratégica de PCAF no SUS e como campo de atuação viria a ser futuramente fortalecida a partir da criação do cargo efetivo de Profissional de Educação Física na saúde, da articulação do serviço com outros pontos da rede de atenção à saúde e do funcionamento do programa em consonância com os princípios e diretrizes do SUS.

Entretanto, é importante mencionar que o arranjo institucional da gestão pública municipal possível à época (década de 1990) para o início do funcionamento do SOE se deu por meio da cessão de professores da Secretaria Municipal de Educação. 
Isso também contribuiu para o pioneirismo do SOE na inserção do Profissional de Educação Física no SUS. Este destaque é importante porque foram estes profissionais com as limitações existentes naquele momento histórico - especialmente no tocante à formação para atuação na saúde pública, o que não era exclusividade da Educação Física (e ainda não é) - os responsáveis pelo início das atividades e pelos primeiros passos para a consolidação do SOE como política pública de saúde.

Nesse mesmo período, ocorre ainda a ampliação do rol de atividades de PCAF ofertadas pelo serviço, que inicialmente se restringia à orientação para a prática de exercícios aeróbios, como corridas e caminhadas. Assim, atividades como a ginástica localizada, ginástica aeróbia e alongamento passaram a ser ofertadas em todos os módulos do SOE. Além dessas, outras práticas, como o Tai Chi Chuan, a ioga e a hidroginástica na praia foram implementadas e ocorrem em pontos específicos do serviço. Nesse contexto, é relevante destacar o pioneirismo e o protagonismo do SOE na promoção das ações de PCAF no SUS e no desenvolvimento das Práticas Integrativas e Complementares em Saúde (PICS), uma vez que a implementação dessas ações é anterior à publicação da Política Nacional de Promoção da Saúde (PNPS) e da Política Nacional de Práticas Integrativas e Complementares no SUS (PNPIC), ambas de 2006. Contudo, estas políticas foram determinantes para a ampliação e o fortalecimento das ações de PCAF e PICS pelo SOE.

Assim, é possível afirmar que neste período (1990 a 2000) houve a consolidação do SOE como política de promoção das PCAF na saúde pública em âmbito municipal, e que este programa se tornou um "embrião" para a implementação de outras iniciativas municipais e também em âmbito nacional pelo Ministério da Saúde.

Apesar de ser vinculado, desde sua concepção, à Semus, é possível identificar, nesta primeira década, que o SOE funcionou com relativa dificuldade de articulação com os demais equipamentos e serviços de saúde, o que foi reconhecido como uma fragilidade do programa, uma vez que este necessitava se configurar como um ponto da rede de serviços de saúde. Em 2004, foi implantado um projeto piloto com a inserção de profissionais de Educação Física do SOE nas equipes multiprofissionais das Unidades Básicas de Saúde (UBS).

Esse projeto teve como objetivo potencializar a articulação do serviço com as equipes de saúde e fortalecer as ações de promoção da saúde e das PCAF na Atenção Primária à Saúde (APS). Assim, a inclusão de profissionais de Educação Física do SOE como membros efetivos das equipes multidisciplinares é anterior à Política Nacional de Atenção Básica à Saúde (PNAB) e à criação do então Núcleo de Apoio à Saúde da Família (Nasf), ocorridos respectivamente em 2006 e 2008.

A publicação da PNPS em 2006 e a instituição da promoção das PCAF como um dos seus eixos prioritários impactaram positivamente o SOE e outros programas municipais, uma vez que foram intensificados os investimentos financeiros e o apoio técnico pelo Ministério da Saúde para o fortalecimento das ações de promoção da saúde e das PCAF no SUS (MALTA et al., 2014a).

Foi nesse mesmo ano que ocorreu a implantação do SOE Móvel, um serviço itinerante que utiliza um veículo adaptado e equipado com recursos humanos e 
materiais para a implementação das ações desenvolvidas pelo SOE em territórios nos quais não existiam módulos físicos instalados. Esse serviço contribui para mapear locais estratégicos à implantação de novos módulos do SOE e, ao mesmo tempo, foi/tem sido importante para o apoio às ações e eventos de promoção da saúde desenvolvidos e/ou apoiados pela Semus.

Data também de 2006 o primeiro concurso público para profissionais de Educação Física na Semus, seguido por outros dois realizados, respectivamente, em 2011 e 2019. A efetivação do concurso possibilitou a recomposição do quadro de profissionais que atuavam nos módulos do SOE, bem como a ampliação do serviço para a inserção de profissionais nas equipes das UBS e nos Centros de Atenção Psicossocial (CAPS) do município. Esse novo cenário foi outro passo importante para o avanço e a consolidação do SOE como programa estratégico do município, o qual exigiu a necessidade de repensar os processos de trabalho instituídos, convocando o grupo de profissionais e gestores para reflexões sobre a atuação do profissional de Educação Física na saúde e para a revisão dos objetivos, organização e funcionamento do serviço.

Nesse contexto, a criação do Nasf, em 2008, e do Programa Academia da Saúde, em 2011, foram marcos determinantes para a reorganização do SOE e contribuíram para fortalecer o funcionamento do serviço em consonância com os princípios, objetivos e diretrizes do SUS. Dessa forma, o SOE influenciou a criação e a produção de materiais técnicos (Caderno Técnico) do Programa Academia da Saúde, mas, ao mesmo passo, foi significativamente reformulado a partir dos princípios, objetivos e diretrizes desse programa. Nesse processo, o SOE foi habilitado como um programa similar ao Academia da Saúde, todavia mantém suas características e objetivos de funcionamento específicos, o que favoreceu sua consolidação na política de saúde do município de Vitória.

Assim, hodiernamente, o SOE, reconhecido como um programa de promoção das PCAF, composto por uma rede de profissionais de Educação Física que atuam em diferentes pontos da Rede de Atenção à Saúde (RAS) do município de Vitória, tem o objetivo de contribuir para a promoção da saúde, prevenção e enfrentamento das doenças e agravos não transmissíveis, tendo em vista a melhoria da qualidade de vida da população.

$\mathrm{Na}$ atualidade, dentre os objetivos específicos do programa, destacam-se os seguintes: a) promover o desenvolvimento das práticas corporais e atividade física como ações de cuidado em saúde; b) contribuir para o aumento da prática de atividade física no lazer e para a redução dos níveis de inatividade física; c) promover e apoiar eventos e ações de educação em saúde; d) contribuir para a ampliação e qualificação dos espaços públicos de lazer de forma a potencializar a sua utilização como forma de inclusão social, enfrentamento das violências e melhoria da qualidade de vida da população; e) fomentar a formação de recursos humanos e o desenvolvimento de pesquisas no âmbito do SUS, visando ao fortalecimento da integração entre ensino, serviço e comunidade. 


\section{A ORGANIZAÇÃO E O FUNCIONAMENTO DO SOE}

O SOE atualmente possui 15 módulos localizados na orla, parques e praças do município, que funcionam diariamente em dois turnos distintos, a priori, matutino e noturno. Os módulos do SOE são equipamentos específicos nos quais atuam os profissionais e se configuram como pontos da RAS. Cada módulo atualmente é vinculado a uma UBS de referência, o que possibilita o desenvolvimento de ações integradas com as equipes de saúde e dos demais equipamentos sociais do território. Além dos módulos do SOE, conforme já destacado, os profissionais do SOE integram as equipes das UBS e dos CAPS, além daqueles que atuam na área técnica e coordenação do programa.

Para atingir os objetivos do SOE, os profissionais desenvolvem ações de cuidado em saúde individuais e coletivas voltadas para os diferentes ciclos de vida (crianças, adolescentes, adultos e idosos), tais como: PCAF, PICS, atividades artísticas e culturais, organização e apoio aos eventos de mobilização comunitária, consultas individuais para avaliação física e orientação ao exercício. Além dessas, destacam-se as ações de educação em saúde e de apoio matricial.

O planejamento e o desenvolvimento das ações desenvolvidas pelo SOE são realizados de forma integrada com as equipes das UBS de referência e são orientados a partir das características epidemiológicas e sociodemográficas do território. A participação social incorporada no planejamento das ações visa ao atendimento dos interesses e necessidades da comunidade. Dessa forma, a atuação dos profissionais do SOE, hodiernamente, orienta-se também pelo que foi sugerido por Loch, Dias e Rech (2019), a saber: atuação coerente com os princípios e diretrizes do SUS; valorização de espaços públicos; participação no planejamento das ações de saúde da Atenção Básica; estímulo e fomento à participação e controle social, dentre outros. Algumas das ações desenvolvidas pelo SOE, inclusive, foram citadas no documento de referência nacional do Programa Academia da Saúde (BRASIL, 2019a).

Especificamente com relação às ações desenvolvidas no SOE, apesar de no âmbito conceitual haver alguma clareza sobre prevenção de doenças e promoção da saúde estabelecidos na literatura (CARVALHO, 2009; CZERESNIA; FREITAS, 2003), é necessário reconhecer que existe, na prática dos serviços de saúde, um "borramento conceitual" (CARVALHO, 2019), não sendo possível estabelecer uma fronteira clara e inequívoca que diferencie as ações nos territórios. De forma geral, as ações buscam seguir referenciais da promoção da saúde, a qual é entendida como um conjunto de estratégias e formas de produzir saúde, no âmbito individual e coletivo, caracterizando-se pela articulação e cooperação intra e intersetorial, pela formação das RAS, buscando articular suas ações com as demais redes de proteção social, com ampla participação e controle social (BRASIL, 2014).

As PCAF, no SOE, se caracterizam como atividades abertas, inclusivas, com poucas barreiras de acesso e visam potencializar o uso e a qualificação dos espaços e equipamentos públicos do município. Tais ações objetivam promover a socialização e a mobilização comunitária, contribuindo para a construção de relações de vínculos, corresponsabilidade e autonomia dos usuários do serviço. Entretanto, as ações também 
almejam a prevenção de doenças e agravos à saúde, já que, em alguns contextos, as PCAF do SOE assumem características e objetivos específicos, como em projetos de prevenção de quedas por idosos, de enfrentamento ao sobrepeso e obesidade, de prevenção e tratamento da hipertensão arterial e diabetes mellitus e em ações voltadas para o controle e redução do uso de tabaco, álcool e outras drogas. Assim, acreditase que as ações do SOE corroboram a afirmativa anterior sobre o borramento, nas práticas, entre promoção da saúde e prevenção de doenças (CARVALHO, 2019).

No que tange ao planejamento e desenvolvimento das PCAF, o serviço se orienta a partir das recomendações globais de atividade física estabelecidas pela OMS (WHO, 2010, 2020), que mostraram uma importante evolução já que os parâmetros abordados em 2010 foram recorrentemente atualizados pela literatura (WARBURTON; BREDIN, 2016, 2017). Além disso, levam em consideração as condições do território e determinantes sociais de saúde específicos envolvidos em cada contexto. Ou seja, são reconhecidas desde as evidências relacionadas aos desfechos em saúde, em especial às DCNT, até o contexto e determinantes sociais da saúde, buscando abarcar a complexidade e multideterminação das PCAF como ações baseadas na compreensão ampliada de saúde.

Assim, o SOE vem buscando estabelecer novos horizontes que extrapolem os benefícios fisiológicos e metabólicos, o que não diminui a importância destes na relação entre as PCAF e a saúde. O SOE reconhece que compreender os sentidos e significados atribuídos às PCAF pelos sujeitos precisa ter a mesma importância que mensurar e quantificar seus benefícios em aspectos biológicos para a saúde (CARVALHO F.; CARVALHO Y., 2018).

Apesar das desejáveis melhorias em componentes da aptidão física e características antropométricas dos usuários do serviço, as PCAF desenvolvidas pelo SOE visam prioritariamente promover aprendizagens e contribuir para o lazer, convivência e socialização entre os usuários do programa. Promover aprendizagens que possam propiciar a autonomia dos sujeitos e o exercício da cidadania requer apresentar todos os elementos constituintes da prática, como história, técnicas, passos, postura, frequência, formas e benefícios, para que o usuário compreenda seu significado, as possibilidades de reconstrução dessas práticas e possa incluí-las em seu cotidiano como um direito, uma escolha (BRASIL, 2019a).

Assim, cabe ressaltar que as PCAF têm mais chances de serem incorporadas pelos indivíduos quando passam a ser uma escolha esclarecida/autônoma, movida pelo prazer, liberdade e gosto pela atividade e não como uma mera obrigação de ordem estética e/ou fisiológica (FRAGA, 2006; PALMA, 2009; PNUD, 2017). É relevante destacar ainda que a escolha não se refere apenas à vontade e/ou esforço pessoal/individual, há fatores que condicionam e determinam o acesso às PCAF, como direito e exercício da cidadania. Desse modo, o "escolher" também está atrelado à possibilidade de o sujeito estranhar e identificar discursos e práticas (midiáticas, científicas e culturais) que buscam governar suas vidas por meio de imperativos morais e apelos comportamentais que estabelecem uma relação de causa e efeito entre atividade física e saúde e/ou sedentarismo e doenças. 
Neste contexto, o SOE amplia suas ações de saúde ao fazer a ruptura de uma defesa inconteste de uma "guerra" ao sedentarismo e às doenças em seu projeto original e primeira década de funcionamento, passando a adotar uma abordagem das PCAF que dá sentido à vida, e que contribui na produção de vínculos sociais e, portanto, na própria aderência das pessoas às ações de PCAF. Entretanto, é necessário ressaltar que esta concepção ampliada das PCAF no campo da saúde não exclui o reconhecimento dos benefícios do SOE e de suas ações para a prevenção de doenças.

Considerando o compromisso assumido com a Organização das Nações Unidades (ONU) pela cidade de Vitória para o Desenvolvimento Sustentável, o planejamento e o desenvolvimento das atividades ofertadas pelo SOE são orientados pelos objetivos e estratégias estabelecidos no Plano de Ação Global de Atividade Física 2018-2030 (WHO, 2018). Esse plano considera a complexidade da promoção da atividade física, bem como a necessidade de uma abordagem sistêmica para que seja possível alcançar os diferentes Objetivos do Desenvolvimento Sustentável (ODS), conforme estabelecido pela Declaração de Bangkok sobre a Atividade Física para a Saúde Global e o Desenvolvimento Sustentável (DECLARAÇÃO..., 2016).

É relevante destacar que as preferências quanto aos tipos de práticas de atividade física variam de acordo com o sexo, idade, escolaridade e região brasileira (WENDT et al., 2019). Portanto, é fundamental que estratégias para promoção ou intervenções com PCAF levem em conta a diversidade de atividades, fornecendo oportunidades de experimentação e/ou foco nas preferências da populaçãoalvo. Além disso, devem considerar ainda as necessidades dos usuários e os determinantes e condicionantes da saúde (CARVALHO, 2016).

Diante desse cenário, o SOE oferta uma ampla diversidade de PCAF coletivas, dentre as quais se destacam: alongamento, ginástica, hidroginástica, danças, circuitos funcionais, caminhada coletiva, capoeira, ioga, esportes adaptados e atividades recreativas. Uma pesquisa realizada pelo Ministério da Saúde avaliou o conhecimento e a participação no SOE. Nesse estudo, os usuários poderiam informar, por ordem de preferência, a participação em mais de uma das atividades ofertadas. Assim, esse levantamento revelou que as atividades mais frequentemente realizadas pelos usuários são o alongamento $(59,2 \%)$, caminhada (31\%) e as ginásticas localizada (40\%) e aeróbica (16,4\%). Constata-se, ainda, que $83,3 \%$ dos participantes do SOE consideram elevada a qualidade das atividades ofertadas (BRASIL, 2010).

As atividades oferecidas pelo SOE assumem características singulares. São adaptadas e/ou criadas pelo profissional do serviço a partir das características do público atendido e das especificidades e demandas de cada território. Por exemplo, a ioga praticada no SOE se constitui por uma prática híbrida. Reconstruída a partir do encontro com a cultura local, ela assume características próprias, diferenciando-se da forma como esta prática acontece em academias e institutos de ioga, possibilitando com que seja denominada de "ioga do SOE" (GOMES, 2018). Essas características do trabalho no SOE o revelam ainda como um espaço de produção e reconstrução de saberes e práticas. 
A seguir, serão apresentadas algumas evidências contidas em estudos acadêmicos e pesquisas sobre as potencialidades das ações desenvolvidas pelo SOE na saúde da população.

\section{AS POTENCIALIDADES DO SOE A PARTIR DA LITERATURA}

Inicialmente, é preciso considerar que, apesar da significativa ampliação de profissionais de Educação Física na saúde pública (SILVA, 2018) e dos reconhecidos benefícios da atividade física para a saúde, ainda são escassas as evidências sobre o impacto de programas de atividade física do SUS (AMORIM et al., 2013; BECKER; GONÇALVES; REIS, 2016), o que constitui um desafio para pesquisadores da área e para os profissionais e gestores do SUS.

Entretanto, foi somente a partir da publicação da PNPS em 2006 e das subsequentes ações de apoio técnico e financeiro pelo Ministério da Saúde que se iniciou, em âmbito nacional, com destaque para o financiamento, a institucionalização das PCAF no SUS (MALTA et al., 2014a). Essas ações atualmente são desenvolvidas, principalmente, por profissionais que atuam no Nasf e no Programa Academia da Saúde (CARVALHO; NOGUEIRA, 2016). Assim, apresentar os resultados de algumas pesquisas sobre o SOE constitui um aspecto relevante para demarcar os 30 anos de funcionamento do serviço e o impacto das suas ações na saúde da população.

O estudo de Rezende (1997) avalia e apresenta os objetivos e características do serviço no final da década de 1990, sendo ressaltado que com vontade política, participação comunitária, criatividade e poucos recursos financeiros, programas como o SOE poderiam ser implantados em todos os municípios do país, integrados ao SUS. Esse apontamento é interessante, visto que, de fato, isso só veio a acontecer com a criação do Programa Academia da Saúde pelo Ministério da Saúde, em 2011, a partir da constituição da Rede Nacional de Atividade Física e do Projeto Guia Útil de Intervenções para Atividade Física no Brasil e na América Latina (KNUTH et al., 2010; PRATT et al., 2010).

O trabalho de Bagrichevsky et al. (2013), que trata das desigualdades sociais em saúde e das PCAF, destaca o SOE como programa pioneiro no setor de saúde, mas apresenta uma análise crítica quanto aos equívocos na definição dos locais selecionados para implantação e expansão dos módulos do serviço, visto que deveriam ser priorizadas as localidades do município com as condições mais precárias para se viver, como forma de reduzir as iniquidades em saúde e de acesso às PCAF.

Apesar disso, Reis et al. (2014) demonstraram que a exposição (conhecimento e participação) ao SOE está consistentemente associada a maiores níveis de prática de atividade física no lazer. A participação no SOE é maior pelas mulheres, idosos, por aqueles com baixo nível educacional e pessoas com morbidades. Esses resultados revelam que, de certa forma, programas públicos de promoção da PCAF no SUS, como o SOE, possibilitam diminuir as iniquidades de acesso às atividades físicas e esportivas no Brasil (PNUD 2017), e ainda se apresentam como política pública eficaz para responder à necessidade de ampliação do acesso à atividade física apontada pela literatura (GUTHOLD et al., 2018; HALLAL et al., 2012). 
Considerando o desafio da saúde pública no enfrentamento das DCNT (MALTA et al, 2014b) e as evidências quanto à importância e benefícios da atividade física para o controle dessas doenças (LEE et al., 2012; PIERCY et al., 2018), essas práticas foram incluídas no Plano de Ações Estratégicas para o Enfrentamento das DCNT no Brasil, 2011-2022 (BRASIL, 2011). O Programa Academia da Saúde foi apontado como uma das principais ações estratégicas para a promoção das PCAF (MALTA et al., 2016).

Nesse contexto, de forma análoga, parece ser relevante destacar resultados de pesquisas sobre o SOE que abordaram essa temática. O trabalho de Venturim e Molina (2005), por exemplo, demonstrou que a participação nas atividades ofertadas pelo SOE está relacionada com a redução de parâmetros hemodinâmicos e antropométricos em adultos. Um outro estudo realizado por Venturim e Cade (2007) revelou que a prática de atividade física ofertada pelo SOE, associada à orientação alimentar e nutricional, evidenciou redução significativa nas variáveis antropométricas (peso corporal, IMC, percentual de gordura corporal e RCQ) e hemodinâmicos (frequência cardíaca e pressão arterial sistólica) de adultos com obesidade. Os resultados dessas pesquisas indicam que as ações ofertadas pelo SOE parecem contribuir para o controle de fatores de risco e o enfrentamento das DCNT.

Com relação à influência do SOE sobre a qualidade de vida, os resultados do trabalho de Ambrosim (2019) indicam que adultos com idade igual ou superior a 40 anos que participam das atividades do SOE apresentam maior pontuação no escore total e em domínios (físico, psicológico e meio ambiente) relacionados à qualidade de vida. Revelam ainda melhores resultados para a saúde nas características antropométricas, perfil bioquímico e na aptidão cardiorrespiratória, quando comparados com o grupo de não adeptos ao programa.

O estudo desenvolvido por Vieira (2019) demonstrou que os idosos participantes do SOE apresentam uma melhor qualidade de vida com maior pontuação nos domínios de capacidade funcional, vitalidade, aspectos sociais e saúde mental. Além disso, a pesquisa constatou um melhor desempenho nos testes de avaliação do equilíbrio postural e da função muscular dos idosos (o que pode contribuir na prevenção de quedas) quando comparados com o grupo de idosos que não participam do serviço.

Sob a perspectiva de que as PCAF precisam ser compreendidas num sentido mais amplo, em que se destaquem o encontro, a convivência, a formação e o fortalecimento de grupos sociais nos territórios, ao criar e/ou aumentar o vínculo entre os sujeitos e destes com os trabalhadores e serviços de saúde, o estudo de Beccalli e Gomes (2014) analisou como os usuários do SOE interagem com o espaço do serviço e o que pensam sobre atividade física e saúde, lembrando que os módulos do SOE se configuram como pontos de encontro da comunidade e que a participação no SOE está atrelada à sensação de bem-estar, relacionada com a produção de sociabilidade e prática de atividade física. Os autores afirmaram que para além da participação nas ações de atividade física, a produção de encontros constitui-se como elemento mobilizador dos indivíduos e, sobretudo, como fator de produção de saúde.

Apesar de o SOE ser considerado um programa consolidado, pioneiro e de referência nacional, tendo sido, inclusive, recentemente reconhecido como uma 
referência exitosa na oferta de atividade física em uma pesquisa de âmbito nacional (SAFE, 2019), ainda existem muitos desafios para o aperfeiçoamento e qualificação do programa. Dentre estes, destacam-se a intersetorialidade e a formação de profissionais para o SUS.

A intersetorialidade tem o potencial de revitalizar as políticas públicas de promoção da saúde, estimulando ações que contemplem os complexos condicionantes do processo saúde-doença, porém ainda se constitui um desafio para o setor saúde (AZEVEDO; PELICIONI; WESTPHAL, 2012). O estudo de Camargo (2017) aponta que, embora o SOE seja uma política de saúde de vanguarda e de referência nacional, esse programa ainda apresenta relações intersetoriais incipientes, classificadas como articulações governamentais horizontais, que é um tipo de intersetorialidade embrionária, mas que abre caminho para a construção de uma política pública com maior alcance e qualidade.

Assumindo, portanto, a intersetorialidade como um desafio para efetivação do SOE como um programa estratégico de promoção da saúde, destaca-se a seguir o desafio relacionado com a formação de profissionais para o SUS, evidenciando como a intersetorialidade e a integração ensino serviço têm contribuído para alguns avanços para a qualificação do serviço.

\section{OS "FIOS” E "DESAFIOS” DO SOE: A QUESTÃO DA FORMAÇÃO}

A formação de profissionais de Educação Física para o SUS vem sendo apontada pela literatura como um dos aspectos necessários para a qualificação da atuação do profissional na saúde coletiva. Tal formação demanda ações das coordenações e dos docentes das Instituições de Ensino Superior (IES) em Educação Física e dos profissionais e gestores dos serviços de saúde (ABENEFS et al., 2016). Nesse sentido, embora as novas Diretrizes Curriculares para os cursos de graduação em Educação Física (BRASIL, 2018), em parte, mostrem-se contraditórias e polêmicas, são apontadas como uma importante oportunidade de aproximação entre os cursos de bacharelado de Educação Física e o SUS (COSTA, 2019).

Diante disso, as ações de integração ensino-serviço com o Curso de Educação Física da UFES têm sido tratadas, em especial nos últimos cinco anos (2015-2020), como uma das prioridades do SOE. Nesse sentido foram estabelecidos "fios" (parcerias) na formação inicial, em projetos de pesquisa na pós-graduação e por meio do Programa de Educação pelo Trabalho para a Saúde (PET-Saúde), bem como em ações como a participação periódica no Congresso Espírito-Santense de Educação Física (Conesef).

Apesar dessas importantes iniciativas e das atuais políticas de formação, ensino e pesquisa nos cursos de graduação e pós-graduação em Educação Física da UFES (FRAGA; CARVALHO; GOMES, 2012), o tema saúde, no curso de Educação Física dessa universidade pública, ainda enfrenta alguns desafios para uma efetiva contribuição na formação de profissionais para o SUS (OLIVEIRA; GOMES, 2019), uma vez que a maior parte das disciplinas curriculares do curso ligadas à saúde ainda 
tem sua base no treinamento desportivo e nos aspectos fisiológicos, bioquímicos e biomecânicos do movimento corporal humano.

O SOE busca também tecer seus "fios" via parcerias em projetos de pesquisas e de extensão com caráter interprofissional, com os cursos de Fisioterapia, Terapia Ocupacional da UFES no Projeto de Prevenção de Quedas em Idosos na Atenção Primária à Saúde e com o Curso de Nutrição dessa mesma instituição, com o Projeto de Orientação Alimentar e Nutricional no SOE (PEREIRA; HARAGUCHI, 2015) e com o Programa de Promoção do Estilo de Vida Saudável na Obesidade - Peso (VENTURIM; CADE, 2007). Recentemente, o SOE tem sido campo de estágio de Educação Física de programas de Residência Multiprofissional em Saúde, configurando uma outra frente de ação para a qualificação de profissionais para o SUS.

No contexto dos desafios da formação de profissionais para o SUS, é necessário destacar que a Educação Permanente em Saúde (EPS) se configura como uma proposta de aprendizagem no trabalho, na qual o aprender e o ensinar se incorporam ao cotidiano das organizações e se baseiam na aprendizagem significativa e na possibilidade de transformar as práticas profissionais (BRASIL, 2007).

Apesar disso, é recente a incorporação das ações de EPS de forma periódica na rotina de trabalho e nas reuniões técnicas do SOE, que passaram, a partir de 2018, a se configurar, também, como um espaço de formação e de socialização de saberes, experiências e práticas. Essa estratégia de transformar esses espaços institucionais em momentos de formação e troca de saberes resultou na realização da I Mostra de Experiências do SOE, realizada em 2019, que teve como tema "o trabalho e as aprendizagens nos territórios de saúde" e que contou com a participação de profissionais e acadêmicos de Educação Física de Vitória e de outros municípios da região metropolitana.

Além desses espaços, a EPS tem sido promovida por meio de cursos e ações de formação pela Escola Técnica e Formação Profissional de Saúde de Vitória Professora Ângela Maria Campos da Silva (ETSUS-Vitória). Essas ações de EPS ocorrem por meio do planejamento ascendente, no qual as necessidades de ações educativas são elaboradas em articulação com os profissionais do serviço, áreas técnicas do SOE e da Semus, em conjunto com a equipe técnica e pedagógica da ETSUS-Vitória.

Há tantos outros desafios ainda mais preocupantes que envolvem o (sub) financiamento do SUS (FUNCIA, 2019), sobretudo a partir da Emenda Constitucional $n^{\circ} .95 / 2016$, que congelou os gastos públicos por 20 anos, constituindo, portanto, uma ameaça para a continuidade, expansão e qualificação de programas de promoção da saúde e das PCAF (LOCH et al., 2017; MALTA et al., 2018) e para a consolidação do SUS (PAIM, 2018).

O novo modelo de financiamento da Atenção Primária à Saúde estabelecido pelo Programa Previne Brasil (BRASIL, 2019b), apesar de colocar o Academia da Saúde como um dos programas estratégicos - embora ainda seja necessário pactuar os indicadores de monitoramento e avaliação de suas ações - , extinguiu 
o financiamento específico para o Núcleo Ampliado de Saúde da Família e Atenção Básica (Nasf AB), assim denominado a partir de 2017. Isso pode ameaçar a continuidade das ações multiprofissionais nos municípios e representar um retrocesso no fortalecimento das ações de PCAF, uma vez que essa equipe tem importância central no desenvolvimento dessas ações e na inserção no SUS (SANTOS; BENEDETTI, 2012; SEUS et al., 2019).

\section{PERSPECTIVAS E CONSIDERAÇÕES FINAIS}

Por fim, é preciso destacar que, após 30 anos de funcionamento, o SOE passa por um momento singular de sua história, no qual os profissionais do serviço e aqueles que atuam na referência técnica e coordenação do programa têm buscado contribuir com as ações de prevenção e enfrentamento da pandemia do Covid-19. Diante desse complexo cenário epidemiológico, as atividades do SOE foram reestruturadas como resposta às necessidades do momento, implicando mudanças na sua organização, o que tem contribuído para o fortalecimento e ampliação das intervenções dos profissionais de Educação Física junto às equipes de saúde.

Nesse contexto, o SOE vem buscando se reinventar mais uma vez. O distanciamento social estabeleceu uma nova demanda do serviço em 2020, exigindo que novas práticas e ferramentas tecnológicas fossem incorporadas para a promoção das PCAF. Diante disso, trabalha-se na produção de um aplicativo para smartphones que objetiva potencializar ações de comunicação social com a população e usuários. Assim, neste momento, no qual o distanciamento social é necessário, videoaulas estão sendo produzidas e disponibilizadas para promover PCAF em casa.

Além disso, vislumbra-se a utilização futura dessa ferramenta como estratégia complementar e permanente para potencializar as ações de PCAF no SUS e para o estreitamento de vínculos com usuários do serviço, tendo em vista o fortalecimento do controle social e das ações de educação em saúde e mobilização da comunidade.

Diante do exposto, a partir da relevância e reconhecimento das PCAF para a promoção e cuidado à saúde, ao longo de 30 anos, o SOE dialogou e incorporou as modificações ocorridas nas políticas de saúde, o que culminou em mudanças em sua organização e funcionamento. Apesar de ser vinculado, desde sua concepção, ao setor da saúde, destacam-se questões, como intersetorialidade incipiente e desafios na formação do profissional de Educação Física. Desses profissionais, por sua vez, exige-se a ampliação de suas possibilidades de compreensão e atuação no SOE para muito além dos aspectos biodinâmicos relacionados à prescrição de exercícios. As evidências trazidas buscam mostrar que o SOE dialoga com os saberes que embasam a Saúde Coletiva: a epidemiologia, as ciências sociais e humanas e, ainda, a política e o planejamento.

Destaca-se, então, o protagonismo e pioneirismo do SOE no campo das PCAF no SUS, ao passo que se reconhece a interação e diálogo com outros programas e práticas que conformaram o serviço na atualidade e que contribuirão para os próximos anos. 


\section{REFERÊNCIAS}

ABENEFS. Manifesto da ABENEFS: alinhando a formação inicial em educação física às necessidades do setor saúde. Revista Brasileira de Atividade Física \& Saúde, v. 21, n. 2, p. 105-109, mar. 2016.

AMBROSIM, Morghana Ferreira. Saúde cardiovascular, nível de atividade física e qualidade de vida de adeptos do Serviço de Orientação ao Exercício. 2019. 76 f. Dissertação (Mestrado em Educação Física) - Centro de Educação Física e Desportos, Universidade Federal do Espírito Santo, Vitória, 2019.

AMORIM, Tales C. et al. Descrição dos programas municipais de promoção da atividade física financiados pelo Ministério da Saúde. Revista Brasileira de Atividade Física \& Saúde, v. 18, n. 1, p. 63-74, jan. 2013.

AZEVEDO, Elaine; PELICIONI, Maria Cecília Focesi; WESTPHAL, Marcia Faria. Práticas intersetoriais nas políticas públicas de promoção de saúde. Physis Revista de Saúde Coletiva, v. 22, n. 4, p. 1333-1356, out./dez. 2012.

BAGRICHEVSKY, Marcos et al. Desigualdades sociais em saúde e práticas corporais: um exercício singular de análise. Saúde e Sociedade, v. 22, n. 2, p.497-510, abr./jun. 2013.

BECCALLI, Michel Binda; GOMES, Ivan Marcelo. Mais que atividade física: usos e entendimentos da saúde e do Serviço de Orientação ao Exercício da Prefeitura Municipal de Vitória entre usuários do serviço. Revista Brasileira de Ciências do Esporte, v. 36, n. 2, p. 26-43, abr./jun. 2014.

BECKER, Leonardo Augusto; GONÇALVES, Priscila Bezerra; REIS, Rodrigo Siqueira. Programas de promoção da atividade física no Sistema Único de Saúde brasileiro: revisão sistemática. Revista Brasileira de Atividade Física \& Saúde, v. 21, n. 2, p. 110-122, mar. 2016.

BRASIL. Ministério da Saúde. Política Nacional de Promoção da Saúde. Brasília: 2018.

BRASIL. Ministério da Saúde. Secretaria de Atenção Primária à Saúde. Departamento de Promoção da Saúde. Programa Academia da Saúde: caderno técnico de apoio à implantação e implementação. Brasília, 2019a.

BRASIL. Ministério da Saúde. Secretaria de Vigilância em Saúde. Avaliação de programas de atividade física no Brasil: uma revisão de evidências em experiências selecionadas. Brasília, 2010.

BRASIL. Ministério da Saúde. Secretaria de Vigilância em Saúde. Plano de ações estratégicas para enfrentamento das doenças crônicas não transmissíveis (DCNT) no Brasil 2011-2022. Brasília, 2011.

BRASIL. Portaria n. ${ }^{\circ}$ 1.996, de 20 de agosto de 2007. Dispõe sobre as diretrizes para a implementação da Política Nacional de Educação Permanente em Saúde. Diário Oficial [da] República Federativa do Brasil, Brasília, DF, v. 144, n. 162, p. 34-38, 2007.

BRASIL. Portaria $n^{\circ} 2.446$, de 11 de novembro de 2014. Redefine a Política Nacional de Promoção da Saúde. Diário Oficial [da] República Federativa do Brasil, Brasília, DF, n. 220, p. 68-70, nov. 2014.

BRASIL. Portaria n².979, de 12 de novembro de 2019. Institui o Programa Previne Brasil. Diário Oficial [da] República Federativa do Brasil, Brasília, DF, n. 220, p. 97. 2019b. 
CAMARGO, Leonardo Perovano. Relações intersetoriais no SOE enquanto política pública que atua com práticas corporais para a população idosa. $2017.85 \mathrm{f}$. Dissertação (Mestrado em Educação Física) - Centro de Educação Física e Desportos, Universidade Federal do Espírito Santo, Vitória, 2017.

CARVALHO, Fabio Fortunato Brasil. A atividade física no discurso governamental brasileiro: análise de alguns meios de comunicação à sociedade. Caderno de Educação Física e Esporte, v.18, n. 2, p.1-6, 2020.

CARVALHO, Fabio Fortunato Brasil. Análise crítica da Carta Brasileira de Prevenção Integrada na Área da Saúde na Perspectiva da Educação Física através do enfoque radical de promoção da saúde. Saúde e Sociedade, v. 18, n. 2, p. 227-236, jun. 2009.

CARVALHO, Fabio Fortunato Brasil. Práticas corporais e atividades físicas na Atenção Básica do Sistema Único de Saúde: ir além da prevenção das doenças crônicas não transmissíveis é necessário. Movimento, v. 22, n. 2, p. 647-658, abr./jun. 2016.

CARVALHO, Fabio Fortunato Brasil. Recomendações de atividade física para a saúde (pública): reflexões em busca de novos horizontes. Arquivos Brasileiros de Ciências da Saúde, v. 44, n. 2, p. 131-137, ago. 2019.

CARVALHO, Fabio Fortunato Brasil; CARVALHO, Yara Maria. Outros... Lugares e modos de "ocupação" da educação física na saúde coletiva/saúde pública. Pensar a Prática, v. 21, n. 4, p. 957-967, out./dez. 2018.

CARVALHO, Fabio Fortunato Brasil, NOGUEIRA, Júlia Aparecida Devidé. Práticas corporais e atividades físicas na perspectiva da promoção da saúde na Atenção Básica. Ciência \& Saúde Coletiva, v. 21, n. 6, p. 1829-1838. jun. 2016.

COSTA, Felipe Ferreira. Novas diretrizes curriculares para os cursos de graduação em Educação Física: oportunidades de aproximações com o SUS? Revista Brasileira de Atividade Física \& Saúde, v. 24, p. 1-4, mar. 2019. DOI: https://doi.org/10.12820/ rbafs. $24 \mathrm{e} 0067$

CZERESNIA, Dina; FREITAS, Carlos Machado (org.). Promoção da saúde: conceitos, reflexões, tendências. Rio de Janeiro: Fiocruz, 2003.

DECLARAÇÃO de Banguecoque sobre a atividade física para a saúde global e desenvolvimento sustentável. Bangkok: ISPAH, 2016. Disponível em: https://ciafel.fade.up.pt/ files_download/bkk_declaration_portuguese_(nov2016)_final.pdf Acesso em: 12 maio 2020.

FRAGA, Alex Branco. Promoção da vida ativa: nova ordem físico-sanitária na educação dos corpos contemporâneos. In: BAGRICHEVSKY et al. A saúde em debate na educação física. Blumenau: Nova Letra, 2006. v. 2, p. 105-120.

FRAGA, Alex Branco; CARVALHO, Yara Maria; GOMES, Ivan Marcelo. Políticas de Formação em Educação Física e Saúde Coletiva. Trabalho, Educação e Saúde, v. 10, n. 3, p. 367-386, nov. 2012.

FUNCIA, Francisco Rózsa. Subfinanciamento e orçamento federal do SUS: referências preliminares para a alocação adicional de recursos. Ciência \& Saúde Coletiva, v. 24, n. 12 , p. 4405-4414, dez. 2019.

GOMES, Lígia Ribeiro e Silva. O yoga no Serviço de Orientação ao Exercício (SOE) em Vitória: ambivalências acerca dos significados atribuídos a uma prática corporal oriental. 2018. 226 f. Tese (Doutorado em Educação Física) - Centro de Educação Física e Desportos, Universidade Federal do Espírito Santo, Vitória, 2018. 
GUTHOLD, Regina et al. Worldwide trends in insufficient physical activity from 2001 to 2016: a pooled analysis of 358 population-based surveys with 1.9 million participants. The Lancet, v. 6, n. 10, p.1077-1086, out. 2018.

HALLAL, Pedro Rodrigues Curi et al. Global physical activity levels: surveillance progress, pitfalls, and prospects. The Lancet, v. 380, p. 247-257, jul. 2012.

KNUTH, Alan Goularte et al. Rede nacional de atividade física do Ministério da Saúde: resultados e estratégias avaliativas. Revista Brasileira de Atividade Física \& Saúde, v. 15, n. 4, p. 229-233, set. 2010.

LEE, I-Min et al. Impact of physical inactivity on the world's major non-communicable diseases. The Lancet, v. 380, n. 9838, p. 219-229, Jul. 2012.

LOCH, Mathias Roberto et al. A revisão da Política Nacional de Atenção Básica e a Promoção da Atividade Física. Revista Brasileira de Atividade Física \& Saúde, v. 22, n. 4, p. 315-318, jul. 2017.

LOCH, Mathias Roberto; DIAS, Douglas Fernando; RECH, Cassiano Ricardo. Apontamentos para a atuação do profissional de educação física na atenção básica à saúde: um ensaio.

Revista Brasileira de Atividade Física \& Saúde, v. 24, p. 1-5, mar. 2019. DOI: https://doi. org/10.12820/rbafs.24e0069

MALTA, Deborah Carvalho et al. Avanços do Plano de Ações Estratégicas para o Enfrentamento das Doenças Crônicas não Transmissíveis no Brasil, 2011-2015. Epidemiologia e Serviço de Saúde, v. 25, n. 2, p. 373-390, abr./jun. 2016.

MALTA, Deborah Carvalho et al. Mortalidade por doenças crônicas não transmissíveis no Brasil e suas regiões, 2000 a 2011. Epidemiologia e Serviço de Saúde, v. 23, n. 4, p. 599608 , out./dez. 2014b.

MALTA, Deborah Carvalho et al. Política Nacional de Promoção da Saúde, descrição da implementação do eixo atividade física e práticas corporais, 2006 a 2014. Revista Brasileira de Atividade Física \& Saúde, v. 19, n. 3, p. 286-299, maio 2014a.

MALTA, Deborah Carvalho et al. O SUS e a Política Nacional de Promoção da Saúde: perspectiva resultados, avanços e desafios em tempos de crise. Ciência \& Saúde Coletiva, v. 23, n. 6, p. 1799-1809, jun. 2018.

OLIVEIRA, Victor José Machado; GOMES, Ivan Marcelo. O tema da saúde na formação inicial em Educação Física em uma universidade pública: reflexividade, agência e estrutura. Movimento, v. 25, p. 2-14, jan./dez. 2019.

PAIM, Jairnilson Silva. Sistema Único de Saúde (SUS) aos 30 anos. Ciência \& Saúde Coletiva, v. 23, n. 6, p.1723-1728, jun. 2018.

PALMA, Alexandre. Exercício físico e saúde; sedentarismo e doença: epidemia, causalidade e moralidade. Motriz, v.15, n.1, p.185-191, 2009.

PEREIRA, Tayenne Dias; HARAGUCHI, Fabiano Kenji. Perfil nutricional dos praticantes de atividades físicas de um módulo do Serviço de Orientação ao Exercício (SOE) do município de Vitória-ES. Revista Brasileira de Nutrição Esportiva, v. 9, n. 52, p. 318-325, jul./ago. 2015.

PIERCY, Katrina L. et al. The physical activity guidelines for Americans. Jama, v. 320, n.19, p. 2020-2028, Nov. 2018. 
PRATT, Michael et al. Project GUIA: A model for understanding and promoting physical activity in Brazil and Latin America. Journal of Physical Activity and Health, v.7, n. 2, p.131-134, 2010.

PNUD - PROGRAMA DAS NAÇÕES UNIDAS PARA O DESENVOLVIMENTO. Relatório de Desenvolvimento Humano Nacional - Movimento é Vida: Atividades Físicas e Esportivas para Todas as Pessoas. Brasília, 2017.

REIS, Rodrigo Siqueira et al. A. Promoting physical activity and quality of life

in Vitoria, Brazil: evaluation of the Exercise Orientation Service (EOS) program. Journal of Physical Activity and Health, v. 11, n. 1, p. 38-44, 2014.

REZENDE, Luciano. SOE-Vitória, ES: sete anos de sucesso com uma ideia simples, eficaz e de baixo custo. Revista Brasileira de Medicina Esportiva, v. 3, n. 3, p. 84-85, jul./set. 1997.

SAFE 2019. Saúde a partir de atividades físicas exitosas. Santa Catarina: UFSC, 2019. Disponível em: http://safe.ufsc.br/equipe/\#sobre. Acesso em: 12 maio 2020.

SANTOS, Sueyla Ferreira da Silva; BENEDETTI, Tania. Cenário de implantação do Núcleo de Apoio a Saúde da Família e a inserção do profissional de educação física. Revista brasileira de Atividade Física \& Saúde, v. 17, n. 3 p. 188-194, jun. 2012.

SEUS, Thamires Lorenzet Cunha et al. Núcleo de Apoio à Saúde da Família: promoção da saúde, atividade física e doenças crônicas no Brasil - inquérito nacional PMAQ 2013. Epidemiologia e Serviço de Saúde, v. 28, n. 2, p. 1-14, jun. 2019.

SILVA, Paulo Sergio Cardoso. Physical Education Professionals in the Unified Health System: an analysis of the Brazilian registry of health institutions between 2013 and 2017. Revista Brasileira de Atividade física \& Saúde, v. 23, p. 1-8, ago. 2018.

VENTURIM, Lara Marina de Vasconcelos Pinho; CADE, Nágela Valadão. Efeitos do programa "Peso" (promoção de estilo de vida saudável na obesidade) sobre variáveis antropométricas, hemodinâmicas e bioquímicas. Revista Brasileira de Atividade Física \& Saúde, v. 12, n. 1, p.19-29, set. 2007.

VENTURIM, Lara Marina de Vasconcelos Pinho; MOLINA, Maria Del Carmen Bisi. Mudanças no estilo de vida após as ações realizadas no serviço de orientação ao exercícioVitória/ES. Revista Brasileira de Atividade Física \& Saúde, v.10, n.2, p.4-16, 2005.

VIEIRA, Leonardo Araújo. Análise do desempenho e da influência da força e potência muscular dos membros inferiores no equilíbrio postural de idosos ativos e sedentários. 2019. 153 f. Dissertação (Mestrado em Educação Física) - Centro de Educação Física e Desportos, Universidade Federal do Espírito Santo, Vitória, 2019.

WARBURTON, Darren E.R.; BREDIN, Shannon S.D. Health benefits of physical activity: a systematic review of current systematic reviews. Current Opinion in Cardiology, v. 32, n. 5, p. 541-556, set. 2017.

WARBURTON, Darren E.R.; BREDIN, Shannon S.D. Reflections on physical activity and health: What should we recommend? Canadian Journal of Cardiology, v. 32, n. 4, p.1-10, mar. 2016.

WENDT, Andrea et al. Preferências de atividade física em adultos brasileiros: resultados da Pesquisa Nacional de Saúde. Revista Brasileira de Atividade Física \& Saúde, v. 24, p. 1-9, mar. 2019. 
WHO - WORLD HEALTH ORGANIZATION. Global action plan to promote physical activity 2018-2030: more active people for a healthier world. Geneva, 2018.

WHO - WORLD HEALTH ORGANIZATION. Global recommendations on physical activity for health. Geneva, 2010.

WHO - WORLD HEALTH ORGANIZATION. Guidelines WHO physical activity and sedentary behaviour for children and adolescents, adults and older adults. Geneva, 2020. 
Abstract: This work analyses the pioneering role played by the Exercise Guidance Service (EGS) in Vitoria, ES, based on its promotion of bodily practices and physical activities (BPPA) in Brazil's Unified Health System (SUS). It is a collective strategy of reflection and writing built by the program's professionals and managers, and partner researchers linked to the field of Physical Education and Public Health. It evaluates and discusses the main changes faced by the EGS over 30 years. It presents how the program has dialogued with health policies and the literature on physical activity and BPPA over three decades. It identifies EGS's potential and challenges as a public health program and highlights BPPA's core role in the SUS. It asserts the importance of coordination and dialogue with other health institutions, policies, programs and practices to improve the services that EGS offers to the population.

Keywords: Health Policy. Motor Activity. Health Systems. Intersectorial Collaboration.

Resumen: El texto analiza el pionerismo del Servicio de Orientación al Ejercicio de Vitória/ES a partir de la promoción de las prácticas corporales y actividades físicas (PCAF) en el Sistema Único de Salud. Se trata de una estrategia colectiva de reflexión tejida por los profesionales, gestores del programa e investigadores colaboradores vinculados a la Educación Física y a la Salud Colectiva. Hace un "balance" y discute las principales transformaciones ocurridas en el Servicio a lo largo de sus 30 años. Presenta como el Servicio viene dialogando, en tres décadas, con las políticas de salud y con la literatura acerca de las PCAF. Identifica potencialidades y desafíos del Servicio como programa de salud pública, destacando su protagonismo en el campo de las PCAF en el SUS. Afirma la importancia de la articulación y diálogo con otras instituciones, políticas, programas y prácticas de salud para la cualificación del servicio ofertado a la población.

Palabras clave: Política de Salud. Actividad Motora. Sistemas de Salud. Colaboración Intersectorial. 


\section{LICENÇA DE USO}

Este é um artigo publicado em acesso aberto (Open Access) sob a licença Creative Commons Atribuição 4.0 Internacional (CC BY 4.0), que permite uso, distribuição e reprodução em qualquer meio, desde que o trabalho original seja corretamente citado. Mais informações em: http://creativecommons.org/licenses/by/4.0

\section{CONFLITO DE INTERESSES}

Os autores declararam que não há conflito de interesses neste trabalho.

\section{CONTRIBUIÇÕES AUTORAIS}

Leonardo Araújo Vieira: Concepção, planejamento, redação, revisão e aprovação da versão final.

Sabrina Barbosa Garcia de Albuquerque: Concepção, planejamento, redação, revisão e aprovação da versão final.

Fábio Olímpio Venturim: Concepção, planejamento, revisão e aprovação da versão final.

Fabio Fortunato Brasil de Carvalho: Planejamento, redação, revisão e aprovação da versão final.

Ueberson Ribeiro Almeida: Planejamento, redação, revisão e aprovação da versão final.

\section{AGRADECIMENTOS}

Agradecemos a todos os profissionais do SOE, gestores da Semus, parceiros da UFES e do Ministério da Saúde e, em especial, aos usuários deste serviço que ao longo desses 30 anos, contribuíram para o fortalecimento do SOE e das PCAF no âmbito do SUS.

\section{FINANCIAMENTO}

O presente trabalho foi realizado sem qualquer apoio financeiro.

\section{COMO REFERENCIAR}

VIEIRA, Leonardo Araújo; ALBUQUERQUE, Sabrina Barbosa Garcia de; VENTURIM, Fábio Olímpio; CARVALHO, Fabio Fortunato Brasil de; ALMEIDA, Ueberson Ribeiro. 30 anos do Serviço de Orientação ao Exercício em Vitória/ES: pioneirismo nas práticas corporais e atividades físicas no Sistema Único de Saúde. Movimento (Porto Alegre), v.26, p. e26086, jan./dez. 2020. Disponível em: https:// seer.ufrgs.br/Movimento/article/view/103142. Acesso em: [dia] [mês abreviado]. [ano]. DOI: https://doi.org/10.22456/1982-8918.103142

\section{RESPONSABILIBADE EDITORIAL}

Alex Branco Fraga*, Elisandro Schultz Wittizorecki, Ivone Job*, Mauro Myskiw*, Raquel da Silveira*

*Universidade Federal do Rio Grande do Sul, Escola de Educação Física, Fisioterapia e Dança, Porto Alegre, RS, Brasil 